\title{
FNC, a Novel Nucleoside Analogue, Blocks Invasion of Aggressive Non-Hodgkin Lymphoma Cell Lines Via Inhibition of the Wnt/ $\beta$-Catenin Signaling Pathway
}

\author{
Yan Zhang', Chen-Ping Wang ${ }^{2}$, Xi-Xi Ding ${ }^{2}$, Ning Wang ${ }^{1}$, Fang Ma ${ }^{1}$, Jin-Hua \\ Jiang1, Qing-Duan Wang ${ }^{1}$, Jun-Biao Chang**
}

\begin{abstract}
Chemotherapy is the primary therapy for malignant lymphoma (ML). However, the clinical outcome is still far from satisfactory. Consequently, an understanding of the mechanism of modulating cancer cell invasion, migration and metastasis is important for the development of more effective chemotherapeutic agents. FNC, 2'- deoxy- 2' - $\beta$ - fluoro -4'- azidocytidine, a novel cytidine analogue, has demonstrated significantly inhibitory effects on proliferation of several non-Hodgkin lymphoma (NHL) cell lines. A previous study indicated that FNC effectively inhibited the growth of Raji and JeKo-1 cells in dose-time dependent effects with $\mathrm{IC}_{50}$ values of $0.2 \mu \mathrm{M}$ and $0.097 \mu \mathrm{M}$, respectively. This study was focused on investigating the anti-invasive properties of FNC on NHL cells and its potential mechanisms of action. Cell adhesion and transwell chamber assays were utilized to investigate the anti-invasive effects of FNC on Raji and JeKo-1 cells. Real-time PCR and Western blotting

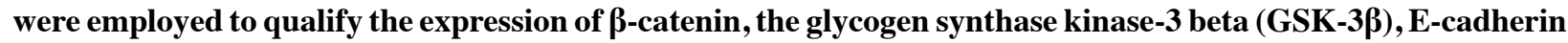
vascular endothelial growth factor (VEGF), matrix metalloproteinase-2 (MMP-2) and matrix metalloproteinase-9 (MMP-9). The results revealed that FNC remarkably inhibited the adhesion, migration and invasion of two human aggressive non-Hodgkin lymphoma cell lines in a dose dependent manner. Furthermore, $\beta$-catenin, MMP-2, MMP-9, VEGF mRNA and protein levels were decreased after FNC treatment, while GSK-3 $\beta$ and E-cadherin increased. Our studies thus provide evidence and a rationale that FNC may offer an effective chemotherapeutic agent by regulating the invasion and metastasis of aggressive non-Hodgkin lymphoma via inhibition of the Wnt/\$-catenin signaling pathway.
\end{abstract}

Keywords: FNC - non-Hodgkin lymphoma - Wnt/3-catenin signaling pathway - MMP-2 - MMP-9 - VEGF

Asian Pac J Cancer Prev, 15 (16), 6829-6835

\section{Introduction}

Malignant lymphoma (ML) belongs to the immune tumor of lymphoid tissue located in and/or external the lymph nodes. According to the pathological types, two main groups of lymphoma have been distinguished. About $90 \%$ of malignant lymphoma is non-Hodgkin lymphoma (NHL), and the rest is called Hodgkin lymphoma (HL). Most common NHL subtype is B-cell non-Hodgkin's lymphoma (B-NHL), including diffuse large B-cell lymphoma (DLBCL), follicular lymphoma (FL), mantle cell lymphoma (MCL), burkitt lymphoma (BL) and other indolent and aggressive histologies. NHL is a worldwide problem and its incidence has been increasing steadily in the past several decades. The growth rate of NHL is about $4 \%-5 \%$ every year and ranks third in all kinds of tumor (Jemal et al., 2011).

NHL has been deemed to the hematologic malignancy with high prevalence and high recurrence. Currently, the treatment of part resection and radiotherapy for NHL could not cure completely in the clinical, in addition to some individual early type. However, chemotherapy and chemoradiotherapy are still main methods to improve the curative effect and prolong patient's survival till now. In the chemotherapy scheme of lymphoma, a classic chemotherapy method, which is still commonly used, is CHOP therapy, which consists of cyclophosphamide, doxorubicin, vincristine, and prednisone. However, using CHOP therapy, patient's survival have historically been poor, particularly in the case of aggressive NHL (Yoh et al., 2014). Since approved by FDA in 1997, the monoclonal CD20 antibody rituximab combinating with CHOP, called R-CHOP therapy, for the treatment of B-NHL represents one satisfactory example in rapid and widespread use (Harrison et al., 2014). Unfortunately, due to the high cost of rituximab, many patients didn't use the recommended dose. Gan GG et al have discovered that there was no demonstrable significant improvement

${ }^{1}$ Academy of Medical and Pharmaceutical Sciences, Zhengzhou University, ${ }^{2}$ School of Pharmaceutical Sciences, Zhengzhou University, ${ }^{3}$ The College of Chemistry and Molecular Engineering, Zhengzhou University, Zhengzhou, China *For correspondence: changjunbiao@zzu.edu.cn 
of patients with DLBCL who were treated with the inadequate dose of rituximab in the R-CHOP therapy (Gan et al., 2014). Although different treatments against this neoplasia exist and spread today, the development of chemoresistance and side effects in tumor cells is the main obstacles to treatment success, which leads to a poor prognosis. Therefore, there is an urgent need to develop more effective anti- lymphoma medication with fewer side effects.

Nucleoside drugs occupy an important position in the treatment of lymphoma. Nucleosides consist of a nucleobase and a ribose or deoxyribose sugar. Because of similar chemical construction to normal metabolic nucleotides, nucleoside drugs can compete with the cellular self-generating nucleotides for incorporation into DNA and RNA strands, eventually leading to block nucleotide synthesis and interfere with the normal metabolism of cells (HodgeTaub et al., 2011).

FNC (2'-deoxy-2'- $\beta$-fluoro-4'-azidocytidine) is a novel nucleoside analogue initially developed as a good antiviral agent in vitro and in vivo (Zheng et al., 2012; Zhou et al., 2012; Wang et al., 2013). At present, FNC has applied for a national patent for invention. The patent number is ZL201010506595.X. The previous research was displayed that FNC had a significantly inhibitory effect on proliferation of several B-NHL cells, showing a good development potential (Wang et al., 2011; Zhang et al., 2013). In order to further research, Raji and JeKo-1 cells which belong to highly invasive B-NHL cells were chosen to explore the anti-invasive effect and mechanism of FNC on aggressive non-Hodgkin's lymphoma.

\section{Materials and Methods}

\section{Reagents}

FNC, which structure is presented in Figure 1, was synthesized by Zhengzhou College of Chemistry and Molecular Engineering, Zhengzhou, China. FNC was dissolved at $6.918 \mathrm{mM}$ in sterile phosphate buffered saline (PBS) as a stock solution, and then further diluted to desired concentrations in cell culture medium for experiments.

\section{Cell line culture}

Raji and JeKo-1 cells were purchased from the Cell Bank of the Chinese Academy of Sciences (Shanghai, China) maintained in Roswell Park Memorial Institute-1640 Medium separately plus $10 \%$ and $20 \%$ fetal bovine serum at $37^{\circ} \mathrm{C}$ in a humidified atmosphere containing $5 \% \mathrm{CO}_{2}$. When the cells reach $70-80 \%$ confluence, they were harvested for cell subculture or subsequent test.

\section{FNC treatment}

All cells were inoculated at a density of $5 \times 10^{6}$ cells per well in 6-well tissue culture plates. Raji cells were treated with $\mathrm{FNC}$ in a final concentration of $0.035 \mu \mathrm{mol} / \mathrm{L}, 0.175$ $\mu \mathrm{mol} / \mathrm{L}, 0.875 \mu \mathrm{mol} / \mathrm{L}$ and $4.375 \mu \mathrm{mol} / \mathrm{L}$; JeKo-1 cells were treated with FNC in a final concentration of 0.0016 $\mu \mathrm{mol} / \mathrm{L}, 0.008 \mu \mathrm{mol} / \mathrm{L}, 0.04 \mu \mathrm{mol} / \mathrm{L}$ and $0.2 \mu \mathrm{mol} / \mathrm{L}$. The control groups were added to their own cell culture medium, respectively. All cells were routinely cultured for 24 hours.

\section{Cell adhesion assay}

The 96-well culture plates were coated with FN $(1 \mu \mathrm{g} /$ well; BD, USA) and bovine serum albumin (BSA, $1 \mathrm{mg}$ / well; Solarbio, China) respectively. The treated cells were processed into single cell suspension and incubated in the 96-well culture plates at a density of $1 \times 10^{5}$ cells/ well. After 1 hour, the unattached cells were removed and washed away by PBS, and MTT colorimetric assay was used to examine the attachment of the cells. The adhesion inhibition rate was calculated: adhesion inhibition rate $(\%)=(1-\mathrm{OD}$ value of experimental group/OD value of control group) $\times 100 \%$.

\section{Migration and invasion assay}

For migration assay, $200 \mu \mathrm{l}$ of treated cells in serum-free RPIM-1640 were seeded on upper migration chambers (24 well plates, $8 \mu \mathrm{m}$ pore size. Corning Incorporated Costar, USA) at a density of $2 \times 10^{5}$ cells /well, and incubated at $37^{\circ} \mathrm{C}$. $600 \mu \mathrm{l}$ of RPIM-1640 containing $10 \%$ fetal bovine serum was added in the lower chamber. Raji cells were incubated for $24 \mathrm{~h}$ and JeKo- 1 cell were incubated for $6 \mathrm{~h}$.

For invasion assay, Matrigel matrix (BD, USA) was dissolved at $4^{\circ} \mathrm{C}$ overnight and then diluted with RPIM1640. The upper chambers were coated with the diluted solution $(100 \mu \mathrm{l} /$ well $)$ and then placed at $37^{\circ} \mathrm{C}$ for $4 \mathrm{~h}$ to solidify the Matrigel matrix. The rest operations were same with migration assay.

The migrating or invading cells were fixed with methanol for 15 minutes, and then stained with $0.1 \%$ crystal violet for 30 minutes. The unpenetrated cells on upper chamber were gently scraped from the surface with cotton swabs. Five visual fields ( $200 \times$ magnification) were selected for each chamber by Image J software (Nikon, Japan) and the number of penetrated cells in these five fields (100×magnification) was counted under a inverted microscope. Mean values of groups were recorded. The migration or invasion inhibition rate was calculated: inhibition rate $(\%)=(1-$ Mean value of experimental group/ Mean value of control group) $\times 100 \%$.

\section{RT-PCR analysis}

Total RNA was extracted with Trizol (ComWin, Beijing, China) from the treated cells. $1 \mu \mathrm{g}$ RNA was used to produce cDNA with the ReverTra Ace (Toyobo, Japan). According to the manufacturer's instructions, $1 \mu \mathrm{l}$ of cDNA was used as PCR template in 12.5 $\mu$ l PCR Mix (ComWin, Beijing, China) with $1 \mu \mathrm{l}$ of each primer and $9.5 \mu \mathrm{ldd}_{2} \mathrm{O}$ ( $25 \mu \mathrm{l}$ reaction volume). $\beta$-actin mRNA level was used for normalization. PCR primer sequences, synthesized by Genewiz of Suzhou, China, are shown as follows. $\beta$-actin: 5'-ATCATGTTTGAGACCTTCAACA-3' (forward); 5'-CATCTCTTGCTCGAAGTCCA-3' (reverse). $\beta$-catenin: 5'- GAAACGGCTTTCAG TTGAGC-3' (forward); 5'-CTGGCCATATCCACC AGAGT-3' (reverse). GSK-3 $\beta$ : 5'-CAGCAAGGT GACAACAGTGG-3' (forward); 5'-GGAACA TAGTCCAGCACCAGA-3' (reverse). E-cadherin: 5'-CTGAAGTGACTCGTAACGAC-3' (forward); 
5'-CATGTCAGCCAGCTTCTTGAAG-3' (reverse). MMP-2: 5'-GGAATGCCATCCCCGATAAC-3' (forward); 5'-CAGCCTAGCCAGTCGGATTT-3' (reverse). MMP-9: 5'-CGTCTTCCCCTTCACTTTCC-3' (forward); 5'-CACAGTAGTGGCCGTAGAAG-3' (reverse). VEGF: 5'-CTTTTCTGCTGTCTTGGGTGC-3' (forward); 5'-ATCGCATCAGGGGCACACAG-3' (reverse). The real-time PCR cycle was performed as follows: $94^{\circ} \mathrm{C}$ for $2 \mathrm{~min}, 40$ cycles of $95^{\circ} \mathrm{C}$ for $30 \mathrm{~s}, 55^{\circ} \mathrm{C}$ for $30 \mathrm{~s}$ and $72^{\circ} \mathrm{C}$ for $45 \mathrm{~s}$, followed by one cycle at $72^{\circ} \mathrm{C}$ for 2 minutes. Amplification products were separated on $3 \%$ agarose gels and photographed with a UV gel imaging system (Kodak, USA). Imagemaster DVS system was used to determine the relative mean gray values (A) of the target product and $\beta$-actin. Expression index (I) of the target product mRNA was calculated: $\mathrm{I}=\mathrm{A}$ product/A $\beta$-actin.

\section{Western blotting analysis}

The treated cells were lysed in RIPA lysis buffer (Biotechwell, China) containing 1\% Protease Inhibitor Cocktail (Biotechwell, China). An equal amount of protein $(30 \mu \mathrm{g})$ was separated by sodium dodecyl sulfate polyacrylamide gel electrophoresis (SDS-PAGE) and transferred onto the poly vinylidene fluoride (PVDF) membrane. After blocking with 5\% skimmed milk, the membrane was probed with primary antibody against $\beta$-catenin and HRP conjugated antirabbit IgG (Proteintech, China). Protein bands were detected with the ECL chemiluminescence Kit (Biotechwell, China). Blots were stripped and photographed. $\beta$-actin level was used to confirm equal protein loading. The anti-GSK-3 $\beta$, anti-E-cadherin, anti-MMP2, anti-MMP9 and anti-VEGF (Biotechwell, China) were used to western blotting analysis on the same way. Imagemaster DVS system was used to determine the relative mean gray values (A) of the target product and $\beta$-actin. Expression index (I) of the target product was calculated: I=A product/A $\beta$-actin.

\section{Statistical analysis}

SPSS version 17.0 was used for data processing. Measurement data presented as the average \pm standard deviation. $p<0.05$ were considered significant. GraphPad Prism 5 (GraphPad Software, USA) was used for graphs. The one-way analysis of the variance (ANOVA) was used to compare adhesion, migration and invasion inhibition rate, mRNA and protein expression of cells between different groups.

\section{Results}

Effect of FNC on the cell adhesion of Raji and JeKo-1 cells FNC could inhibit Raji and JeKo-1 cells attaching to FN. The adhesion inhibition rates of Raji cells treated with FNC at $0.035 \mu \mathrm{mol} / \mathrm{L}, 0.175 \mu \mathrm{mol} / \mathrm{L}, 0.875 \mu \mathrm{mol} / \mathrm{L}$ and $4.375 \mu \mathrm{mol} / \mathrm{L}$ for 24 hours were $(33.50 \pm 0.48) \%$, $(39.18 \pm 0.75) \%,(48.93 \pm 0.24) \%$ and $(57.63 \pm 1.05) \%$ respectively. Compared with the control group, the adhesion of cells treated with FNC was significantly inhibited $(\mathrm{n}=3, p<0.05)$. The adhesion inhibition rates of JeKo-1 cells treated with FNC at $0.0016 \mu \mathrm{mol} / \mathrm{L}$,
$0.008 \mu \mathrm{mol} / \mathrm{L}, 0.04 \mu \mathrm{mol} / \mathrm{L}$ and $0.2 \mu \mathrm{mol} / \mathrm{L}$ for 24 hours were $(13.78 \pm 0.54) \%,(27.16 \pm 1.41) \%,(35.39 \pm 0.99) \%$ and $(51.84 \pm 0.67) \%$ respectively. Compared with the control group, the adhesion of cells treated with FNC was significantly inhibited $(n=3, p<0.05)$ (Figure 2$)$.

FNC inhibited the migration and invasion of Raji and JeKo-1 cells

The migrating and invading cells in chambers were presented in Figure 3A which indicates that FNC treatment inhibited the migration and invasion of Raji and JeKo-1 cells.

The migration inhibition rate in Raji cells was $(13.47 \pm 1.51) \%,(37.97 \pm 0.93) \%,(41.83 \pm 1.08) \%$ and $(54.97 \pm 0.96) \%$; the invasion inhibition rate in Raji cells was $(20.07 \pm 1.16) \%,(36.07 \pm 0.65) \%,(46.50 \pm 1.03) \%$ and $(55.83 \pm 1.10) \%$, respectively. Compared with the control group, the adhesion of cells treated with FNC was significantly inhibited $(n=3, p<0.05)$ (Figure 3B).

The migration inhibition rate in JeKo-1 cells was $(14.69 \pm 0.93) \%,(21.56 \pm 0.41) \%,(33.65 \pm 1.51) \%$ and (40.18 \pm 1.07$) \%$; the invasion inhibition rate in JeKo-1 cells was $(10.98 \pm 0.57) \%,(16.31 \pm 1.04) \%,(29.35 \pm 0.76) \%$ and $(44.77 \pm 0.99) \%$, respectively. Compared with the control group, the adhesion of cells treated with FNC was significantly inhibited $(\mathrm{n}=3, p<0.05)$ (Figure $3 \mathrm{C})$.

Effect of FNC on the mRNA level of $\beta$-catenin, GSK-3 $\beta$, E-cadherin, MMP-2, MMP-9 and VEGF expression from Raji and JeKo-1 cells

To investigate the probable anti-invasive mechanism

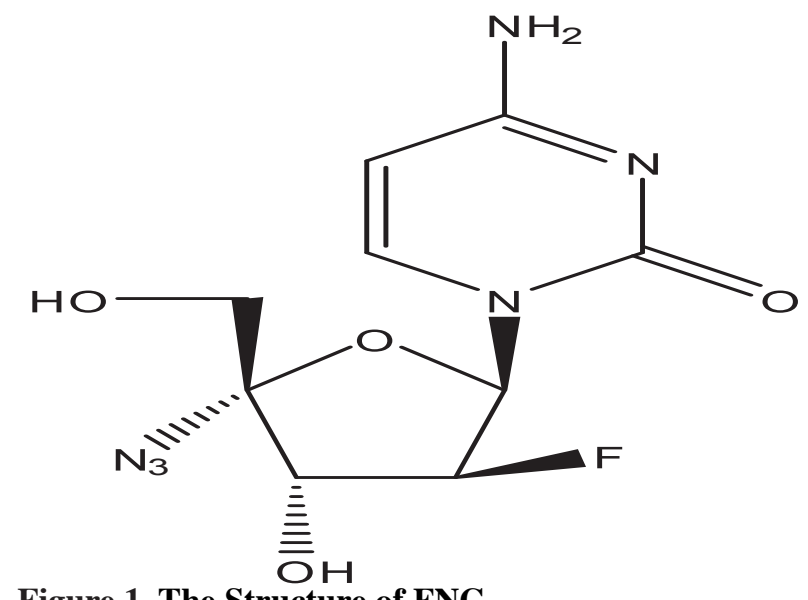

Figure 1. The Structure of FNC
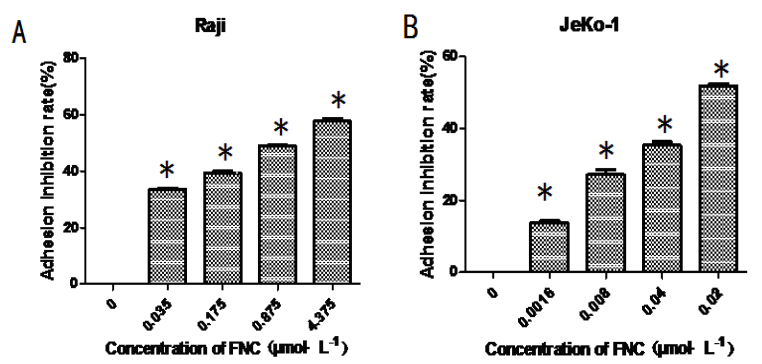

Figure 2. Adhesion Assay to Fibronectin (FN) of Raji and JeKo-1 Cells. A: Adhesion inhibition rates of Raji cells indiciting significant difference compare to control group. B: Adhesion inhibition rates of JeKo- 1 cells indiciting significant difference compare to control group $(* p<0.05 v s$ the control group) 
of FNC on Raji and JeKo-1 cells, we analyzed the level of $\beta$-catenin, GSK-3 $\beta$, E-cadherin, MMP-2, MMP-9 and VEGF mRNA and protein expression. Up-regulation of GSK-3 $\beta$, E-cadherin and down-regulation of $\beta$-catenin, MMP-2, MMP-9, VEGF mRNA expression was observed in Raji and JeKo-1 cells treated with FNC for 24 hours (Figure 4A). The results revealed that the effect of FNC on $\beta$-catenin, GSK-3 $\beta$, E-cadherin, MMP-2, MMP-9 and VEGF mRNA expression varied significantly in different FNC concentration $(p<0.05)$ (Figure 4B; Figure 4C).

Effect of FNC on the protein level of $\beta$-catenin, GSK-3 $\beta$, E-cadherin, MMP-2, MMP-9 and VEGF expression from Raji and JeKo-1 cells

The results of Western blotting are analogous in RT-
PCR experiments. Up-regulation of GSK-3 $\beta$, E-cadherin and down-regulation of $\beta$-catenin, VEGF, MMP-2, MMP-9 protein expression was observed in Raji and JeKo-1 cells treated with FNC for 24 hours (Figure $5 \mathrm{~A})$. The results revealed that the effect of FNC on $\beta$-catenin, GSK-3 $\beta$, E-cadherin, MMP-2, MMP-9 and VEGF mRNA expression varied significantly in different FNC concentration and kept a consistency with mRNA expression $(p<0.05)$ (Figure 5B; Figure 5C).

\section{Discussion}

Nucleoside analogues have been in clinical use to treat various malignancies for almost 50 years. Over the past decade, this family, including purine analogues, adenosine
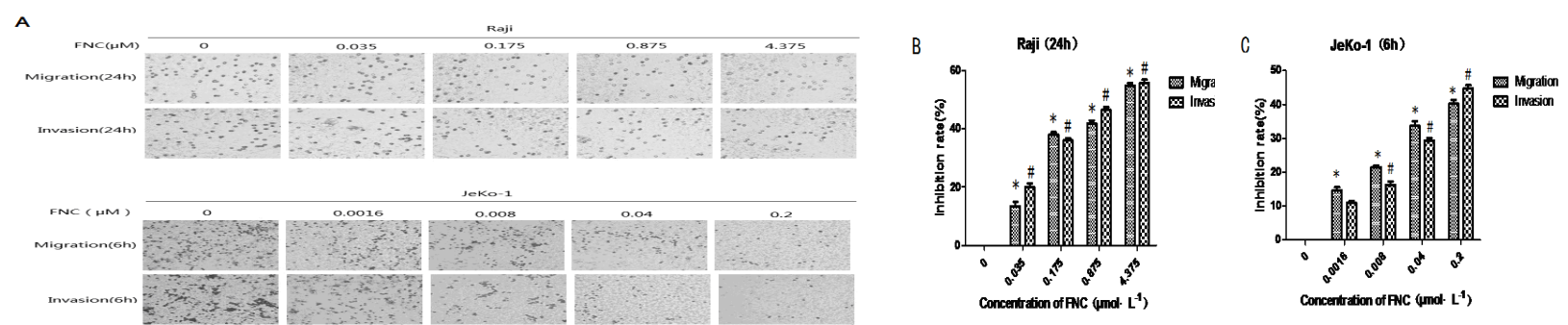

Figure 3. The Anti-Migrate and Anti-Invasive Effect for FNC on Raji and JeKo-1 Cells. A) The cells were photographed under phase-contrast microscopy (original magnification $\times 200$ )). B) The inhibition rates of FNC for Raji cells were quantified. C: The inhibition rates of FNC for JeKo-1 cells were quantified. ( ${ }^{*} p<0.05 v s$ the migration control group; \#p $<0.05 v s$ the invasion control group)
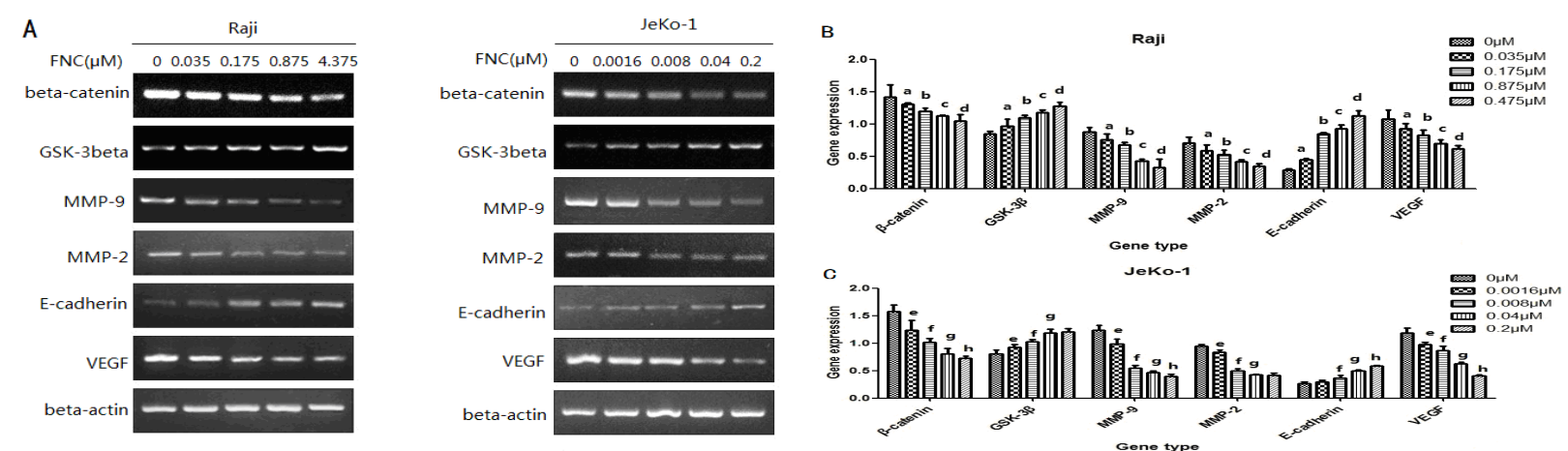

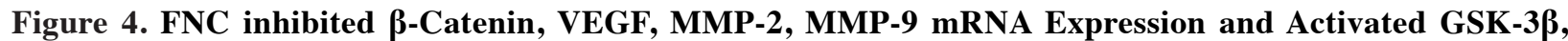
E-Cadherin mRNA Expression in Raji and JeKo-1 Cells. A) The expression of genes was photographed. B) The changes of genes expression in Raji cells (a: $v s 0 \mu \mathrm{M}$ group, $p<0.05$; b: $v s 0.035 \mu \mathrm{M}$ group, $p<0.05$; c: $v s 0.175 \mu \mathrm{M}$ group, $p<0.05$; d: $v s$ $0.875 \mu \mathrm{M}$ group, $p<0.05$ ). C) The changes of genes expression in JeKo-1 cells (e: $v s 0 \mu \mathrm{M}$ group, $p<0.05$ : f: $v s 0.0016 \mu \mathrm{M}$ group, $p<0.05$; g: vs $0.008 \mu \mathrm{M}$ group, $p<0.05$; h: vs $0.04 \mu \mathrm{M}$ group, $p<0.05$ )
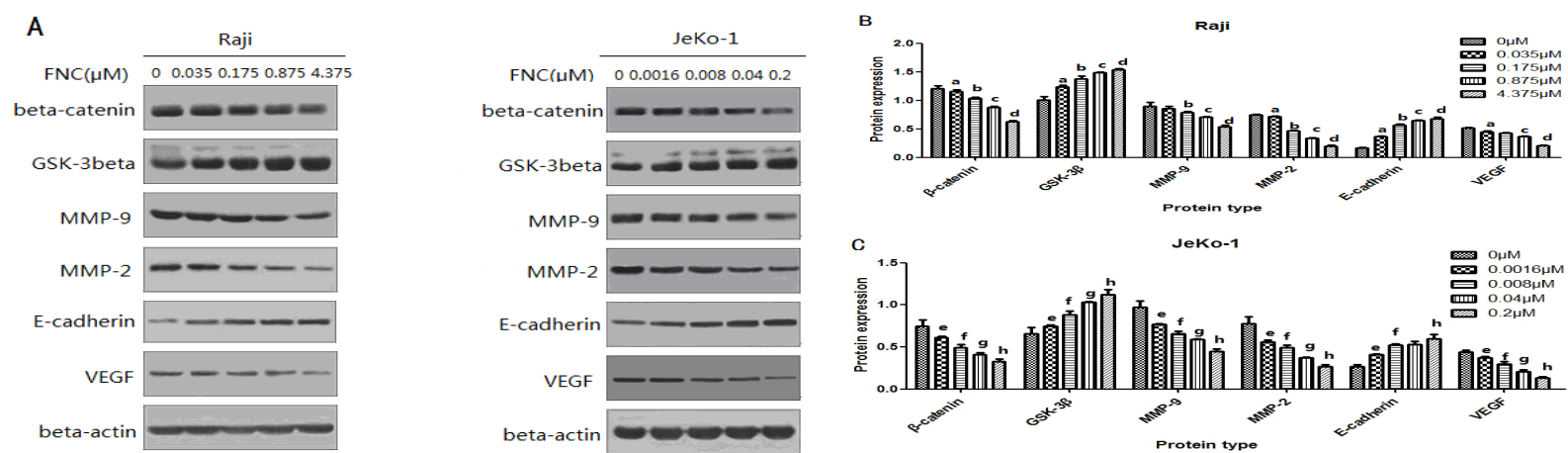

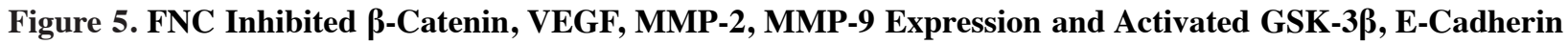
Expression in Raji and JeKo-1 Cells. A) The expression of proteins was photographed. B) The changes of proteins expression in Raji cells (a: $v s 0 \mu \mathrm{M}$ group, $p<0.05$; b: $v s 0.035 \mu \mathrm{M}$ group, $p<0.05$; c: $v s 0.175 \mu \mathrm{M}$ group, $p<0.05$; d: $v s 0.875 \mu \mathrm{M}$ group, $p<0.05$ ). C) The changes of proteins expression in JeKo-1 cells (e: $v s 0 \mu \mathrm{M}$ group, $p<0.05$; f: $v s 0.0016 \mu \mathrm{M}$ group, $p<0.05$; g: $v s 0.008 \mu \mathrm{M}$ group, $p<0.05$; h: vs $0.04 \mu \mathrm{M}$ group, $p<0.05$ ) 
analogues, cytidine analogues, guanosine analogues and uracil analogues, still possesses strong potential (Jordheim et al., 2013). Because nucleosides are the metabolic precursors of nucleotides, nucleoside analogues can affect nucleotide metabolism (Tuncbilek et al., 2012). Because of similar structure, nucleoside analogues can block nucleotide synthesis by incorporation into DNA to inhibit cellular processes, including cell growth and division (Liu et al., 2013).

FNC, a novel cytidine analogue, is an excellent substrate for deoxycytidine kinase and is phosphorylated with higher efficiency than deoxycytidine (Klumpp et al., 2008). In previous study, we demonstrated the significant anti-tumor activity of FNC on high aggressive NHL cells, and FNC effectively inhibited the growth of Raji and JeKo- 1 cells in dose-time dependent effect with $\mathrm{IC}_{50}$ values $0.2 \mu \mathrm{M}$ and $0.097 \mu \mathrm{M}$, respectively. Thus, FNC could be a new agent for NHL patients.

NHL has three different pathological types: indolent NHL, aggressive NHL and high invasive NHL. The treatment will become more difficult when NHL becomes more aggressive. In clinical, BL and MCL are aggressive forms of B cell non-Hodgkin lymphoma with systemic lymph node enlargement and extensive extranodal lesions resulting in a poor prognosis. In order to further clarify the effect and mechanism of FNC on aggressivity of Raji and JeKo- 1 cells, we carried on this topic research.

Invasion and metastasis is an important biological character of malignant tumors. The process is quite complicated and involves in multiple molecular mechanisms, including the adhesion, migration and invasion. Tumor cells were free after proliferation in primary lesion and then adhere to the basement membrane. Adhesion plays a very important role in keeping the shape of a cell, modulating cell division and movement, and it is also the initial step of tumor cell invasion (Xiao et al., 2011). After attachment to the basement membrane, tumor cells could release a variety of substrate protease to degrade the basement membrane. That makes tumor cells to move away from the original focus and invade into adjacent tissues, blood vessel or lymphatic vessel, leading to the formation of metastases in the distance.

In this study, we found that FNC could significantly inhibit the adhesion of Raji and JeKo- 1 cells to FN and also obviously suppress the migration and invasion of Raji and JeKo- 1 cells, in a dose dependent manner (Figure 2 and Figure 3), indicating that one of the important mechanism for FNC with a good resistance to malignant lymphoma is inhibiting invasion and metastasis of tumor cells.

With the deepening of the mechanism research, promoting the invasion and metastasis of tumor cells involves in various factors, including the continuous activation of $\mathrm{Wnt} / \beta$-catenin signaling pathway (Yuan et al., 2012; Rao et al., 2013), abnormal regulation of E-cadherin signal mechanism (Bae et al., 2013), abnormal degradation of cell matrix in tumor microenvironment and excessive release of angiogenine (Mehner et al., 2014; Saarelainen et al., 2014). They interact with each other to enhance the invasion and metastasis of tumor cells.

The contribution of aberrant Wnt/ $\beta$-catenin signaling, an ancient and evolutionarily conserved signaling pathway, has been shown to play a fundamental role in the development and promotion of cancer. The main components of this signaling pathway are including Wnt proteins, the frizzled proteins (Fzls), low density lipoprotein receptor related protein5/6 (LRP5/6), GSK-3 $\beta$, disheveled proteins (Dsh), Axin, adenomatous polyposis coh (APC), $\beta$-catenin, T-cell factor/lymphocyte enhancer factor (TCF/LEF) and its downstream target genes (SaitoDiaz et al., 2013). Whether Wnt/ $\beta$-catenin signaling pathway is activated or not is directly determined by the concentration of $\beta$-catenin. In normal circumstances, free $\beta$-catenin always maintains in a low-density because of degradation by ubiquitin and proteasome. Only E-cadherin- $\beta$-catenin complex exists in the cytoplasm. Abnormally high expression of $\beta$-catenin causes aberrant $\mathrm{Wnt} / \beta$-catenin signaling, leading to prompt occurrence and development of a wide variety of cancers. In addition, GSK- $3 \beta$, an upstream gene of $\beta$-catenin, has the function of negatively regulating $\mathrm{Wnt} / \beta$-catenin signaling pathway. $\beta$-catenin is regulated through phosphorylation by the destruction complex in which GSK-3 $\beta$ is one of the key components. Phosphorylated $\beta$-catenin by GSK- $3 \beta$ is then ubiquitinylated, resulting in its proteasomal degradation (Gelebart et al., 2008). Thus, GSK-3 $\beta$ and $\beta$-catenin are two key components in $\mathrm{Wnt} / \beta$-catenin signaling pathway. When $\mathrm{Wnt} / \beta$-catenin signaling pathway is activated via some kind of factors, Wnt proteins combine to their respective receptors composed of Fzls and LRP5/6s, and then trigger Dish activated and GSK-3 $\beta$ inactivated. When GSK- $3 \beta$ is inactivated, $\beta$-catenin is released from the destruction complex and allowed to accumulate in the cytoplasm and migrate to the nucleus. In the nucleus, accumulated $\beta$-catenin interacting with the TCF/LEF family of DNA-bound transcription factors activates downstream target genes, mainly oncogenes, cell cycle regulators, VEGF and matrix degrading enzymes, affecting the occurrence and development of cancers (Leow et al., 2014).

Cadherins are a family of transmembrane glycoproteins that play an indispensable role in intercellular adhesion. One group of members of this family, E-cadherin molecules, is expressed predominantly on epithelial cells, where they promote the adhesion reaction in mutual epithelial cells or between cells and cell matrix, mediate cell connections, maintain the integrity of the epithelial tissue and the polarity of cells, and involve in cell differentiation. The structure of E-cadherin is mainly composed of intracellular domain, across membrane domain and extracellular domain. The cytoplasmic domain of E-cadherin binds to $\beta$-catenin; the clustering of E-cadherin- $\beta$-catenin complexes on adjacent cells then leads to promote cell-cell adhesions and maintain the polarity of the same cells (Canel et al., 2013). The loss of E-cadherin and the resulting suppression, or weakening has been regarded as a crucial step in tumor invasion and metastasis. Thus, E-cadherin gene is considered as an important suppressor of tumor metastasis. However, activated $\mathrm{Wnt} / \beta$-catenin altering the E-cadherin- $\beta$-catenin complex expression, is significantly associated with the invasiveness of tumor cells (Kim et al., 2013). The absence of E-cadherin leads to an accumulation of $\beta$-catenin with 
LEF1 in the nucleus and activation of a Wnt reporter. On the contrary, increasing expression of E-cadherin could reduce the transcription of $\beta$-catenin to suppress the activity of $\mathrm{Wnt} / \beta$-catenin signaling pathway (Heuberger et al., 2010).

Complex changes in the tumor microenvironment (TME) play an important role in regulating the growth, invasion and metastasis of tumor. MMP-2 and MMP-9, belonging to gelatin enzymes type of matrix metalloproteinases, mainly hydrolyze gelatin and type IV collagenase which is the main component of extracellular matrix (ECM). Studies have confirmed that MMP-2 and MMP-9 has played a key role in the degradation of ECM in the NHL patients (Kossakowska et al., 1999). Thus, MMP2 and MMP-9 are two key molecules in the tumor invasion. Moreover, MMPs are the down-stream genes of $\beta$-catenin. Studies have shown that $\mathrm{Wnt} / \beta$-catenin signaling pathway promotes the expression of MMPs through regulating COX-2, leading to promote invasion and metastasis of tumor (Kim et al., 2009; Peng et al., 2011).

Blood vessels are considered as important channels for tumor cells to invade outward. Lymphoma metastasis is dependent on angiogenesis too. VEGF, an important regulatory angiogenic molecule, directly affects the mitogen of vascular endothelial cell to stimulate proliferation, migration of endothelial cell and increase vascular permeability. VEGF could induce endothelial cells to secrete cathepsin, including metal matrix protein enzymes, leading to degradate ECM and mediate endothelial cell migration. In addition, MMP-2 can not only degradate ECM, but also accelerate the growth of vascular endothelial cells from gap to the outside to gradually extend vascular bud in the process of new blood vessels (Herszenyi et al., 2012). Thus, metal matrix protein enzymes can promote the expression of VEGF and the formation of tumor blood vessels to promote the invasiveness of tumor cells.

In this work, high expression of $\beta$-catenin and low expression of GSK-3 $\beta$ in negative control groups showed that Wnt $/ \beta$-catenin signaling pathway was aberrantly activated in Raji and JeKo-1 cells. Down-regulated expression of $\beta$-catenin and up-regulated expression of GSK-3 $\beta$, E-cadherin with the treatment of FNC for 24 hours supposed that FNC suppressed the aberrant activation of Wnt/ $\beta$-catenin signaling pathway, enhanced Cadherin proteins signal to reinforce cell adhesion, and down-regulated the expression of MMP-2, MMP-9 and VEGF which are the downstream target genes of Wnt/ $\beta$ catenin signaling pathway, leading to inhibit the invasion of Raji and JeKo-1 cells. On the other hand, FNC may also inhibit the invasion of Raji and JeKo-1 cells by directly affecting MMP-2, MMP-9 and VEGF. From these results, we can conclude that FNC may have anti- invasive effect on aggressive non-Hodgkin lymphoma through multiple targets. Therefore, FNC could be a promising agent for future treatment of aggressive non-Hodgkin lymphoma.

\section{Acknowledgements}

This work was supported by the National Natural Science Foundation of China (NO. 21172202) and the
Scientific and technological project of Henan Province, China (NO. 112101310007).

\section{References}

Bae GY, Choi SJ, Lee JS, et al (2013). Loss of E-cadherin activates EGFR-MEK/ERK signaling, which promotes invasion via the ZEB1/MMP2 axis in non-small cell lung cancer. Oncotarget, 4, 2512-22.

Canel M, Serrels A, Frame MC, et al (2013). E-cadherin-integrin crosstalk in cancer invasion and metastasis. J Cell Sci, 126, 393-401.

Gan GG, Subramaniam R, Bee PC, et al (2014). Impact of inadequate doses of rituximab in the treatment of diffuse large B cell lymphoma in Malaysian patients. Asian Pac J Cancer Prev, 15, 1703-6.

Gelebart P, Anand M, Armanious H, et al (2008). Constitutive activation of the Wnt canonical pathway in mantle cell lymphoma. Blood, 112, 5171-9.

Harrison AM, Thalji NM, Greenberg AJ, et al (2014). Rituximab for non-Hodgkin's lymphoma: a story of rapid success in translation. Clin Transl Sci, 7, 82-6.

Herszenyi L, Hritz I, Lakatos G, et al (2012). The behavior of matrix metalloproteinases and their inhibitors in colorectal cancer. Int J Mol Sci, 13, 13240-63.

Heuberger J, Birchmeier W (2010). Interplay of cadherinmediated cell adhesion and canonical Wnt signaling. Cold Spring Harb Perspect Biol, 2, 2915.

Hodge LS, Taub ME, Tracy TS. (2011). Effect of its deaminated metabolite, 2', 2'-difluorodeoxyuridine, on the transport and toxicity of gemcitabine in HeLa cells. Biochem Pharmacol, 81, 950-6.

Jemal A, Bray F, Center MM, et al (2011). Global cancer statistics. CA Cancer J Clin, 61, 69-90.

Jordheim LP, Durantel D, Zoulim F, et al (2013). Advances in the development of nucleoside and nucleotide analogues for cancer and viral diseases. Nat Rev Drug Discov, 12, 447-64.

Kim, H, Yoo SB, Sun P, et al (2013). Alteration of the E-cadherin/ beta-catenin complex is an independent poor prognostic factor in lung adenocarcinoma. Korean J Pathol, 47, 44-51.

Kim S, Kim SH, Hur SM, et al (2009). Silibinin prevents TPAinduced MMP-9 expression by down-regulation of COX-2 in human breast cancer cells. J Ethnopharmacol, 126, 252-7.

Klumpp K, Kalayanov G, Ma H, et al (2008). 2'-deoxy-4'-azido nucleoside analogs are highly potent inhibitors of hepatitis $\mathrm{C}$ virus replication despite the lack of 2'-alpha-hydroxyl groups. J Biol Chem, 283, 2167-75.

Kossakowska AE, Edwards DR, Prusinkiewicz C, et al (1999). Interleukin-6 regulation of matrix metalloproteinase (MMP2 and MMP-9) and tissue inhibitor of metalloproteinase (TIMP-1) expression in malignant non-Hodgkin's lymphomas. Blood, 94, 2080-9.

Leow PC, Bahety P, Boon CP, et al (2014). Functionalized curcumin analogs as potent modulators of the Wnt/betacatenin signaling pathway. Eur J Med Chem, 71, 67-80.

Liu W, Zhang L, Zhou H, et al (2013). Synthesis of novel nucleoside analogue phosphorothioamidate prodrugs and in vitro anticancer evaluation against RKO human colon carcinoma cells. Nucleosides Nucleotides Nucleic Acids, 32, 161-73.

Mehner C, Hockla A, Miller E, et al (2014). Tumor cell-produced matrix metalloproteinase 9 (MMP-9) drives malignant progression and metastasis of basal-like triple negative breast cancer. Oncotarget, 5, 2737-49.

Peng ZH, Wan DS, Li LR, et al (2011). Expression of COX-2, MMP-2 and VEGF in stage II and III colorectal cancer and the clinical significance. Hepatogastroenterology, $\mathbf{5 8}$, 
369-76.

Rao C, Lin SL, Wen H, et al (2013). Crosstalk between canonical TGF-beta/Smad and Wnt/beta-catenin signaling pathway. Zhejiang Da Xue Xue Bao Yi Xue Ban, 42, 591-6.

Saarelainen SK, Staff S, Peltonen N, et al (2014). Endoglin, VEGF, and its receptors in predicting metastases in endometrial carcinoma. Tumour Biol, 35, 4651-7.

Saito-Diaz K, Chen TW, Wang X, et al (2013). The way Wnt works: components and mechanism. Growth Factors, 31, $1-31$.

Tuncbilek M, Guven EB, Onder T, et al (2012). Synthesis of novel 6- (4-substituted piperazine-1-yl)-9- (beta-D-ribofuranosyl) purine derivatives, which lead to senescence-induced cell death in liver cancer cells. $J$ Med Chem, 55, 3058-65.

Wang J, Wang X, Gao C, et al (2013). The pyrimidine analog FNC inhibits cell proliferation and viral protein synthesis in HTLV1 infected cells. Mol Med Rep, 7, 1656-60.

Wang Q, Liu X, Wang Q, et al (2011). FNC, a novel nucleoside analogue inhibits cell proliferation and tumor growth in a variety of human cancer cells. Biochem Pharmacol, 81, 848-55.

Xiao R, Zhao Y, Wang LJ, et al (2011). Effects of Roundabout 5 on adhesion, invasion and potential motility of human tongue carcinoma Tb cells. Chin Med J, 124, 2367-71.

Yoh KA, Lee HS, Park LC, et al (2014). The prognostic significance of elevated levels of serum ferritin before chemotherapy in patients with non-Hodgkin lymphoma. Clin Lymphoma Myeloma Leuk, 14, 43-9.

Yuan JB, Yang LY, Tang ZY, et al (2012). Down-regulation of EZH2 by RNA interference inhibits proliferation and invasion of ACHN cells via the Wnt/beta- catenin pathway. Asian Pac J Cancer Prev, 13, 6197-201.

Zhang Y, Cheng X, Huang G, et al (2013). Effect of new nucleoside analogue FNC on proliferation, apoptosis and expressions of Bcl-6, PRDM1, C-myc in cell line Raji. $J$ Zhengzhou University, 4, 450-4.

Zheng L, Wang Q, Yang X, et al (2012). Antiviral activity of FNC, 2'-deoxy-2'-beta-fluoro-4'-azidocytidine, against human and duck HBV replication. Antivir Ther, 17, 679-87.

Zhou Y, Zhang Y, Yang X, et al (2012). Novel nucleoside analogue FNC is effective against both wild-type and lamivudine-resistant HBV clinical isolates. Antivir Ther, 17, 1593-9. 